Research Article

\title{
Upregulation of Potassium Voltage-Gated Channel Subfamily J Member 2 Levels in the Lungs of Patients with Idiopathic Pulmonary Fibrosis
}

\author{
Jong-Uk Lee, ${ }^{1}$ Hun Soo Chang, ${ }^{2}$ Chang An Jung, ${ }^{2}$ Ryun Hee Kim, ${ }^{2}$ Choon-Sik Park, \\ and Jong-Sook Park $\mathbb{1}^{1}$ \\ ${ }^{1}$ Genome Research Center and Division of Allergy and Respiratory Medicine, Soonchunhyang University Bucheon Hospital, \\ Bucheon, Republic of Korea \\ ${ }^{2}$ Department of Interdisciplinary Program in Biomedical Science Major, Soonchunhyang Graduate School, Bucheon, \\ Republic of Korea
}

Correspondence should be addressed to Jong-Sook Park; newstart1221@naver.com

Received 16 August 2019; Accepted 23 January 2020; Published 26 February 2020

Academic Editor: Michael Roth

Copyright $\odot 2020$ Jong-Uk Lee et al. This is an open access article distributed under the Creative Commons Attribution License, which permits unrestricted use, distribution, and reproduction in any medium, provided the original work is properly cited.

Background. Fibroblast dysfunction is the main pathogenic mechanism underpinning idiopathic pulmonary fibrosis (IPF). Potassium voltage-gated channel subfamily J member $2(\mathrm{KCNJ} 2)$ plays critical roles in the proliferation of myofibroblasts and in the development of cardiac fibrosis. Objectives. This study aimed to evaluate the role of KCNJ2 in IPF. Methods. KCNJ2 mRNA expression was measured using real-time PCR in fibroblasts from IPF patients and normal controls (NCs). Protein concentrations were measured by ELISA in bronchoalveolar lavage (BAL) fluid obtained from NCs $(n=30)$, IPF $(n=84)$, nonspecific interstitial pneumonia (NSIP; $n=9$ ), hypersensitivity pneumonitis (HP; $n=8)$, and sarcoidosis $(n=10)$. Results. KCNJ2 mRNA levels were significantly higher in fibroblasts from IPF $(n=14)$ than those from NCs $(n=10, p<0.001)$. KCNJ2 protein levels in BAL fluid were significantly higher in IPF $(6.587$ [1.441-26.01] ng/mL) than in NCs $(0.084$ [0.00-0.260] ng/ $\mathrm{mL}, p<0.001)$, NSIP (0.301 [0.070-5.059] ng/mL, $p=0.006)$, HP $(0.365$ [0.000-3.407] ng/mL, $p=0.02)$, and sarcoidosis $(0.170$ [0.057-1.179] $\mathrm{ng} / \mathrm{mL}, p=0.001)$. Receiver operating characteristic curves showed a clear difference between the IPF and NCs according to the KCNJ2 protein level (area under the curve $=0.893$ ). The KCNJ2 protein cutoff level determined from the curves $(0.636 \mathrm{ng} / \mathrm{mL})$ showed a $90.0 \%$ specificity and $83.3 \%$ sensitivity in distinguishing IPF from NCs. Conclusion. KCNJ2 may participate in the development of IPF, and its protein level may be a candidate diagnostic and therapeutic molecule for IPF.

\section{Introduction}

Idiopathic interstitial pneumonia is a group of lung diseases of unknown etiology characterized by complex interactions among cells involved in inflammation and fibrosis. Idiopathic pulmonary fibrosis (IPF) is a well-known type of idiopathic interstitial pneumonia with a poor prognosis [1]. Although the disease course of IPF varies [2,3], it is usually progressive in cases with irreversible pulmonary fibrosis. Several lines of evidence suggest that genetic and epigenetic mechanisms play roles in the development and prognosis of IPF [4]. Among the genetic factors, the expression of
MUC5B, SFTPC, SFTPA2, RTEL1, TERT, and $h T R$ is altered in the lungs of IPF patients compared with healthy subjects and those with other lung diseases [5]. Recently, global gene expression levels in lung tissues were compared to identify genes involved in the complex mechanism of IPF [6-9]. We also identified 178 of the total of 15,020 genes that were differentially expressed in lung fibroblasts from IPF patients and controls [10]. Among these genes, the mRNA expression of potassium voltage-gated channel subfamily J member 2 (KCNJ2) was 18.6-fold higher in IPF than control subjects.

As an inward rectifier potassium channel, KCNJ2 transports potassium ions $\left(\mathrm{K}^{+}\right)$more readily into the cell 
than out of the cell [11-14]. Because KCNJ2 is predominantly expressed in skeletal and cardiac muscle cells [15], this channel plays a physiological role in maintaining the resting potentials and controlling the excitability of cells $[16,17]$. KCNJ2 knockdown decreased the $\mathrm{Ca}^{2+}$ influx and proliferative activity of atrial fibroblasts [18]. Exposure to low levels of $\mathrm{K}^{+}$, which induces hyperpolarization, enhanced the proliferation and survival of myofibroblasts; in contrast, exposure to elevated $\mathrm{K}^{+}$caused depolarization [19]. Clinically, KCNJ2 is involved in the development of Andersen syndrome and atrial fibrillation. Additionally, this channel appears to be essential for maintaining the cellular functions of oligodendrocytes, astrocytes, epithelial cells, and fibroblasts [20-23]. Furthermore, phosphatidylinositol 4,5bisphosphate, which maintains the KCNJ2 channel in the open state $[16,24,25]$, is involved in cellular proliferation and angiogenesis in IPF [26]. Thus, KCNJ2 is expected to play a role in the development of IPF. However, KCNJ2 expression has not been evaluated in patients with IPF. Therefore, in the present study, we evaluated the relationships between KCNJ2 expression and the clinical characteristics of IPF by measuring KCNJ2 protein and mRNA levels in fibroblasts and bronchoalveolar lavage (BAL) fluid from normal controls (NC), patients with IPF, and patients with other interstitial lung diseases, including nonspecific interstitial pneumonia (NSIP), hypersensitivity pneumonitis (HP), and sarcoidosis.

\section{Materials and Methods}

We followed the methods provided by Lee et al. [10].

2.1. Study Subjects. Lung tissues and BAL fluids from patients with diffuse interstitial lung diseases were obtained from the biobank of Soonchunhyang University Hospital, Bucheon, Korea (schbc-biobank-2015-013) after approval of the study protocol by the Ethics Committee of Soonchunhyang University Hospital (SCHBC-IRB-2015-08-25). Informed written consent for study participation and sample donation was obtained from each subject. The diagnostic criteria for IPF, HP, NSIP, and sarcoidosis were based on an international consensus statement [1, 27-30]. All subjects were examined by a physician to obtain their medical history and undergo chest X-ray, high-resolution chest computed tomography (HRCT), and pulmonary function tests. None of the IPF patients had any evidence of underlying collagen vascular diseases according to laboratory tests and clinical symptoms. IPF was diagnosed by the presence of usual interstitial pneumonia (UIP) patterns in the pathological specimens (surgical IPF) and/or by HRCT in patients who did not undergo surgical lung biopsy (clinical IPF). Two pathologists examined each slide independently after being informed of the patients' sex, age, and HCRT results.

Pathological recognition of the NSIP pattern included two major aspects: (1) exclusion of other patterns of interstitial lung diseases and (2) categorization of the histological features according to the ATS/ERS 2002 classification
$[30,31]$ and the modified histological definition of the NSIP pattern [32]. HP was diagnosed by the presence of clinical symptoms compatible with nonnecrotizing granulomatous bronchiolocentric pneumonitis [27]. The diagnosis of sarcoidosis was based on histological evidence of noncaseating granuloma and compatible clinical images [28, 29]. HP and sarcoidosis were diagnosed after excluding other diseases with similar histological profiles. Biopsy tissues were subjected to acid-fast bacilli and Gömöri methenamine silver staining to verify the absence of microorganisms and fungi. Serial DLCO and FVC were measured, and the annual rate of FVC decline was estimated as follows: (last FVC-baseline FVC)/baseline FVC/follow-up years. The NCs exhibited no respiratory symptoms, as determined by a screening questionnaire [32], and had a predicted FEV1 and FVC $>80 \%$ and normal chest radiograms.

The total GAP score was calculated using the method suggested by Ley et al. [33]. The following four clinical variables were examined to determine the GAP score: sex $(0$ points for female, 1 point for male), age (0-2 points), FVC $\%$ (0-2 points), and DLCO \% (0-3 points). We divided the patients into eight groups according to their GAP score: group $0(n=4)$, group $1(n=12)$, group $2(n=16)$, group 3 $(n=15)$, group $4(n=15)$, group $5(n=12)$, group $6(n=6)$, and group $7(n=4)$. DLCO was not measured in eight subjects with IPF and thus was excluded from the determination of their GAP scores.

2.2. Reverse Transcription Polymerase Chain Reaction (RTPCR) and Real-Time PCR Detection of KCNJ2 mRNA of Cultured Fibroblasts. Lung fibroblasts were cultured from the surgical lung specimens of 14 IPF patients and 10 subjects who underwent surgery for stage I or II lung cancer, as described previously [34].

The extracted RNA was treated using the Turbo DNAFree $^{\mathrm{TM}}$ Kit (Ambion). The resulting total RNA $(1 \mu \mathrm{g})$ was suspended in diethylpyrocarbonate-treated water with $0.5 \mu \mathrm{g}$ $10 \mathrm{mM}$ dNTPs and oligodeoxythymidine, heated at $65^{\circ} \mathrm{C}$ for $5 \mathrm{~min}$, and then cooled on ice. PCR amplification was performed for 30 cycles $\left(5 \mathrm{~min}\right.$ at $94^{\circ} \mathrm{C}, 30 \mathrm{~s}$ at $94^{\circ} \mathrm{C}, 30 \mathrm{~s}$ at $60^{\circ} \mathrm{C}$, and $30 \mathrm{~s}$ at $72^{\circ} \mathrm{C}$ ) with a final extension at $72^{\circ} \mathrm{C}$ for $7 \mathrm{~min}$. The PCR mixture $(20 \mu \mathrm{L})$ contained $1 \mu \mathrm{g} \mathrm{cDNA}, 10 \mu \mathrm{L}$ $2 \times$ Power SYBR Green PCR Master Mix (Applied Biosystems, Foster City, CA, USA), and $1 \mu \mathrm{L} 10$ pmol forward and reverse primers. The following primer sequences were used: KCNJ2 (sense) $5^{\prime}$-CACTCCATGTCCCCATGCTC- $3^{\prime}$ and (antisense) 5'-CCGCTACAGCATCGTCTCTT-3'; $\beta$-actin (sense) $5^{\prime}$-GGACTTCGAGCAAGAGATGG-3' and (antisense) $5^{\prime}$-AGCACTGTGTTGGCGTACAG-3'. The PCR products were separated on a $1.0 \%$ agarose gel containing ethidium bromide in Tris-borate EDTA buffer at $100 \mathrm{~V}$ for $40 \mathrm{~min}$ and visualized under ultraviolet light. The KCNJ2 band intensities were normalized to those of $\beta$-actin. Real-time PCR was executed using the StepOne ${ }^{\mathrm{TM}}$ Real-Time PCR System (Applied Biosystems) in a two-step procedure: denaturation at $95^{\circ} \mathrm{C}$ for $15 \mathrm{~s}$ and $60^{\circ} \mathrm{C}$ for $1 \mathrm{~min}$, followed by melting at $95^{\circ} \mathrm{C}$ for $15 \mathrm{~s}, 60^{\circ} \mathrm{C}$ for $1 \mathrm{~min}$, and $95^{\circ} \mathrm{C}$ for $15 \mathrm{~s}$. Expression levels were calculated using the $2^{-\Delta \Delta \mathrm{CT}}$ method 
[35] and expressed as the relative fold change after normalization to PPIA.

\subsection{Enzyme-Linked Immunosorbent Assay (ELISA) of KCNJ2} in BAL Fluid. This study was performed using the KCNJ2 ELISA for the diagnosis in IPF between October 2015 and January 2018 . The BAL procedure was performed in the lung segments exhibiting greatest disease involvement on HRCT under no immunosuppressive therapy, or in the right middle lobe of the NCs, as described previously [2, 36-38]. Differential cell counts were performed among 500 cells from BAL fluid placed on slides prepared using a cytocentrifuge and Diff-Quik staining. The total cell count was performed using a hemocytometer. Cells were removed from the supernatants by centrifugation $(500 \mathrm{~g}, 5 \mathrm{~min})$, and the supernatants were stored at $-80^{\circ} \mathrm{C}$. The $\mathrm{KCNJ} 2$ protein level was measured by ELISA (MyBioSource, San Diego, CA, USA) according to the manufacturer's recommendations. The lower limit of detection was $0.625 \mathrm{ng} / \mathrm{mL}$, and values below this limit were set to 0 . The interassay and intra-assay coefficients of variation were less than $15 \%$.

2.4. Statistical Analysis. Receiver operating characteristic (ROC) analysis was performed, and the area under the ROC curve (AUC) and cutoff values were determined using MedCalc statistical software. Comparisons of the KCNJ2 level between groups were performed using the Kruskal-Wallis test and post hoc Mann-Whitney $U$ test. Correlations between the KCNJ2 level and other parameters were analyzed using Spearman's correlation coefficient. The data are presented as medians with $25 \%$ and $75 \%$ quartiles for variables with a skewed distribution or as mean$s \pm$ standard error of the mean for variables with a normal distribution. The data were analyzed using SPSS v. 20.0.

\section{Results}

3.1. Clinical Characteristics of Study Groups. Fibroblast cultures were obtained from 14 IPF patients and 10 NCs who underwent lung surgery, and the clinical information and laboratory data of these subjects were summarized in our previous study [10]. BAL fluid samples were obtained from patients with IPF $(n=84)$, NSIP $(n=9)$, hypersensitivity pneumonitis $(n=8)$, and sarcoidosis $(n=10)$, and the clinical characteristics of these patients are summarized in Table 1. Patients with IPF had significantly higher macrophage, neutrophil, and eosinophil counts in the BAL fluid and lower FVC and FEV1 values than NCs $(p<0.05)$. The IPF group included 40 clinical and 44 surgical IPF patients, and their clinical characteristics were also summarized in our previous study [10].

3.2. KCNJ2 mRNA Levels in Cultured Fibroblasts. Fibroblasts from the lungs of the 14 IPF patients and $10 \mathrm{NCs}$ were used to evaluate KCNJ2 levels. The KCNJ2 mRNA level in fibroblasts was two-fold higher in the IPF patients than in the NCs according to RT-PCR $(p<0.001$; Figures $1(\mathrm{a})$ and $1(\mathrm{~b}))$ and real-time PCR $(p=0.03$; Figures $1(\mathrm{c})$ and $1(\mathrm{~d}))$. The KCNJ2 mRNA levels of our previously measured by transcriptomic analysis in the 12 subjects [10] showed a strong correlation with those determined by real-time PCR $(r=0.755$, $p=0.005$; Figure $1(\mathrm{e}))$.

3.3. KCNJ2 Protein Levels in BAL Fluids. The protein levels of KCNJ2 in BAL fluids, measured by ELISA, were significantly higher in IPF patients $(6.587[1.441-26.01] \mathrm{ng} / \mathrm{mL})$ than those in NCs $(0.084[0.000-0.260] \mathrm{ng} / \mathrm{mL} ; p<0.001)$, NSIP patients $(0.301[0.070-5.059] \mathrm{ng} / \mathrm{mL} ; p=0.006)$, HP patients $(0.365[0.000-3.407] \mathrm{ng} / \mathrm{mL} ; p=0.02)$, and sarcoidosis patients $(0.170 \quad[0.057-1.179] \mathrm{ng} / \mathrm{mL} ; \quad p<0.001)$ (Figure 2(a)). The ROC curve showed a clear difference between the IPF patients and NCs (AUC $=0.893$, Figure 2(b)) and between the IPF patients and groups with all other interstitial lung disease $(n=27)(\mathrm{AUC}=0.822$, Figure 2(c)). The cutoff KCNJ2 level of $0.636 \mathrm{ng} / \mathrm{mL}$ determined from the ROC curve showed a $90.0 \%$ specificity and an $83.3 \%$ sensitivity for distinguishing between the NCs and IPF patients. The cutoff KCNJ2 level of $1.795 \mathrm{ng} / \mathrm{mL}$ exhibited an $81.48 \%$ specificity and a $72.62 \%$ sensitivity for distinguishing between the patients with IPF and those with other interstitial lung diseases.

3.4. Comparison of KCNJ2 Protein Levels according to IPF Clinical Characteristics. There were no differences in the KCNJ2 protein levels according to the GAP stage (stage I vs. II vs. III: 6.692 [1.244-26.59] ng/mL vs. 3.791 [1.676-17.10] $\mathrm{ng} / \mathrm{mL}$ vs. $19.34 \quad[3.142-42.10] \mathrm{ng} / \mathrm{ml}$; Supplemental Figure 1(a)), smoking status (SM vs. ES vs. NS: 7.765 $[1.151-25.42] \mathrm{ng} / \mathrm{mL}$ vs. 6.375 [1.483-16.28] ng/mL vs. 6.692 [1.489-28.83] ng/mL; Supplemental Figure 1(b)), sex (males vs. females: $6.375[1.398-25.13] \mathrm{ng} / \mathrm{mL}$ vs. 6.692 [1.489-27.03] ng/mL; Supplemental Figure 1(c)), or presence of arrhythmia (arrhythmia vs. no arrhythmia: $2.582[0.596-3.232] \mathrm{ng} / \mathrm{mL}$ vs. $1.208[0.239-3.975] \mathrm{ng} / \mathrm{mL}$; Supplemental Figure 2).

\section{Discussion}

In this study, we demonstrated that KCNJ2 gene and protein expression was significantly increased in fibroblasts and BAL fluids, respectively, from patients with IPF compared with $\mathrm{NCs}$ and patients with other interstitial lung diseases. Additionally, the cutoff KCNJ2 protein level of $0.636 \mathrm{ng} / \mathrm{mL}$ exhibited $90.0 \%$ specificity and $83.3 \%$ sensitivity for diagnosing IPF. To the best of our knowledge, this is the first evidence suggesting that KCNJ2 expression is related to the development of IPF and that the KCNJ2 protein level may be a surrogate biomarker for differentially diagnosing IPF among other interstitial lung diseases. ROC curves showed a clear difference between the IPF patients and those with NSIP, HP, or sarcoidosis at a cutoff KCNJ2 protein level of $1.795 \mathrm{ng} / \mathrm{mL}$.

We did not evaluate the cellular source of KCNJ2 production in BAL fluid, which is one of the limitations of the present study. Thus, it is uncertain whether fibroblasts or 
TABLE 1: Clinical characteristics of the study subjects who underwent bronchoalveolar lavage.

\begin{tabular}{|c|c|c|c|c|c|}
\hline Items & Normal controls & IPF & NSIP & HP & Sarcoidosis \\
\hline No. & 30 & 84 & 9 & 8 & 10 \\
\hline Age (year) & $55(35-72)$ & $63.41(59-75)$ & $54.18(39-70) \dagger$ & $44.58(27-63) \dagger$ & $41.90(27-68) \dagger$ \\
\hline Sex (male/female) & $17 / 13$ & $51 / 33$ & $3 / 6$ & $3 / 5$ & $6 / 4$ \\
\hline $\begin{array}{l}\text { Smoke (NS/ES/ } \\
\text { SM/ND) }\end{array}$ & $7 / 9 / 14$ & $19 / 25 / 36$ & $3 / 2 / 1 / 2$ & $5 / 1 / 1 / 1$ & $5 / 2 / 3 / 0$ \\
\hline $\begin{array}{l}\text { Follow-up } \\
\text { duration (year) }\end{array}$ & ND & $3.8(1.7-6.5)$ & ND & ND & ND \\
\hline Survival/Death & $26 / 4$ & $25 / 59$ & $7 / 2$ & $8 / 0$ & $10 / 0$ \\
\hline FVC (\% pred.) & $115.00(106.25-132.25)$ & $67.0(52.0-80.00)^{*}$ & $62.00(50.50-82.00)^{*}$ & $59.50(54.50-81.50)^{*}$ & $86.50(72.50-99.25)^{*} \dagger$ \\
\hline FEV1 (\% pred.) & $104.00(88.48-121.25)$ & $89.50(59.0-93.00)^{*}$ & $64.00(56.50-94.00)^{*} \dagger$ & $71.50(58.00-92.00)^{*} \dagger$ & $98.00(80.50-106.25)$ \\
\hline DLCO (\% pred.) & $86(79-110)$ & $57.00(46.0-71.00)$ & $62.00(57.00-73.00)$ & $68.50(56.25-85.00)$ & $89.00(81.50-98.00) \dagger$ \\
\hline dFVC (\%/year) & NA & $-6.8(-13.6-2.8)$ & NA & NA & NA \\
\hline $\begin{array}{l}\text { BAL total cell } \\
\text { count }(\mathrm{x} 104 / \mathrm{mL})\end{array}$ & $3.46 \pm 0.82$ & $7.72 \pm 2.42^{*}$ & $18.56 \pm 3.91^{*}$ & $13.03 \pm 3.78^{*}$ & $9.13 \pm 3.29^{*}$ \\
\hline $\begin{array}{l}\text { Macrophages } \\
(\text { x104/mL) }\end{array}$ & $3.02 \pm 0.43$ & $6.12 \pm 1.78^{*}$ & $12.91 \pm 3.57^{*}$ & $7.85 \pm 2.38^{*}$ & $7.26 \pm 3.61^{*}$ \\
\hline $\begin{array}{l}\text { Neutrophils (x104/ } \\
\mathrm{mL} \text { ) }\end{array}$ & $0.31 \pm 0.047$ & $1.87 \pm 0.15^{*}$ & $2.31 \pm 1.42^{*}$ & $3.42 \pm 2.36^{*}$ & $0.48 \pm 0.18^{*}$ \\
\hline $\begin{array}{l}\text { Eosinophils (x104/ } \\
\mathrm{mL} \text { ) }\end{array}$ & $0.02 \pm 0.01$ & $0.51 \pm 0.08^{*}$ & $0.72 \pm 0.16^{*}$ & $0.21 \pm 0.18^{*}$ & $0.12 \pm 0.11$ \\
\hline $\begin{array}{l}\text { Lymphocytes } \\
\text { (x104/mL) }\end{array}$ & $0.02 \pm 0.01$ & $0.27 \pm 0.15$ & $2.32 \pm 0.12 * \dagger$ & $2.40 \pm 0.11^{*} \dagger$ & $2.44 \pm 0.21^{*} \dagger$ \\
\hline
\end{tabular}

IPF: Idiopathic pulmonary fibrosis; NSIP: Nonspecific interstitial fibrosis; HP: Hypersensitivity pneumonitis CS/ES/NS: current-smokers/ex-smokers/ never-smokers; ND: not determined; dFVC (\%): annual decline rate of FVC. Difference in patient characteristics and pulmonary function test, shown as median (IQR), among the controls, IPF, NSIP, HP, and sarcoidosis groups was calculated with Kruskal-Wallis analysis of variance and Mann-Whitney $U$ test as post hoc test. BAL cell numbers, shown as mean \pm standard error of the mean, among the five groups were compared using one-way ANOVA analysis of variance with Tukey's honestly significant difference test as post hoc test. Significance: compared with normal controls: ${ }^{*} P<0.05$, compared with IPF: $\dagger P<0.05$.

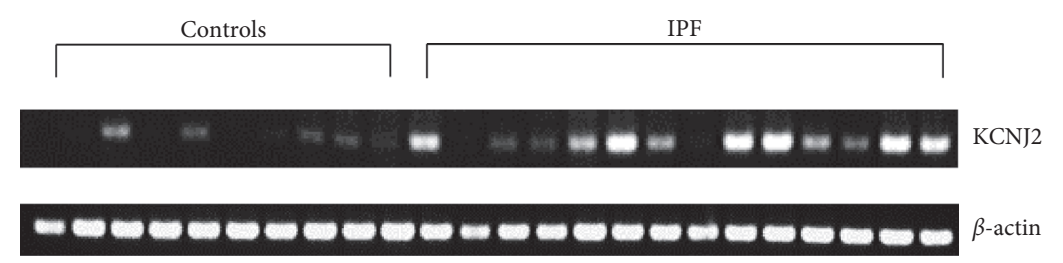

(a)

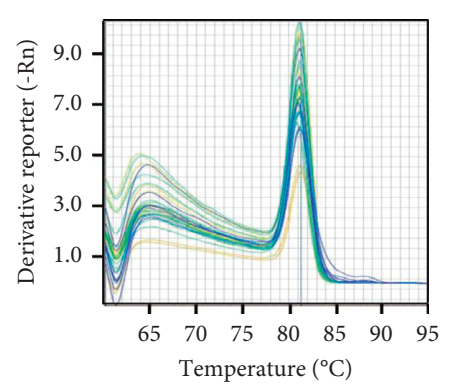

(c)

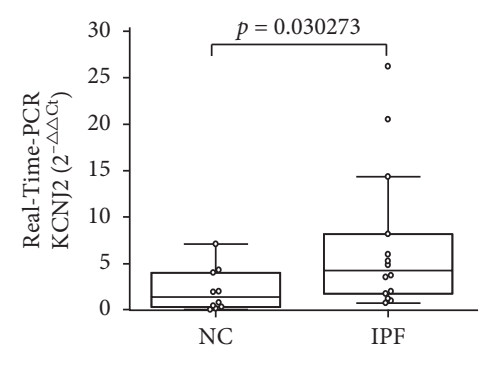

(d)

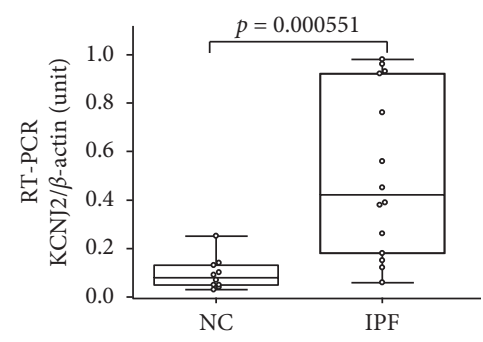

(b)

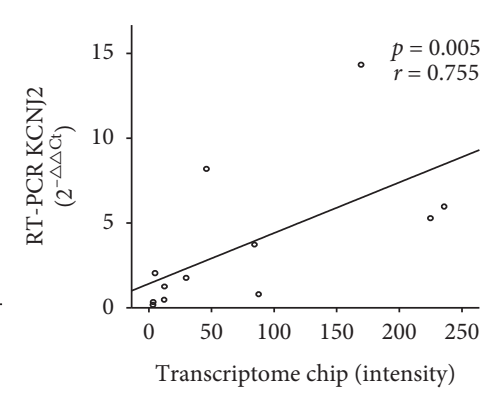

(e)

Figure 1: KCNJ2 mRNA levels in lung fibroblasts from 14 IPF patients and 10 NCs. (a) RT-PCR and (b) densitometric analysis of the KCNJ2 band intensities after normalization to those of $\beta$-actin. (c) Real-time PCR melt curves and (d) relative gene expression levels. (e) Correlations of the KCNJ2 mRNA levels $(n=12)$ between transcriptome chips [10] and real-time PCR. The data are presented as medians with $25 \%$ and $75 \%$ quartiles. 


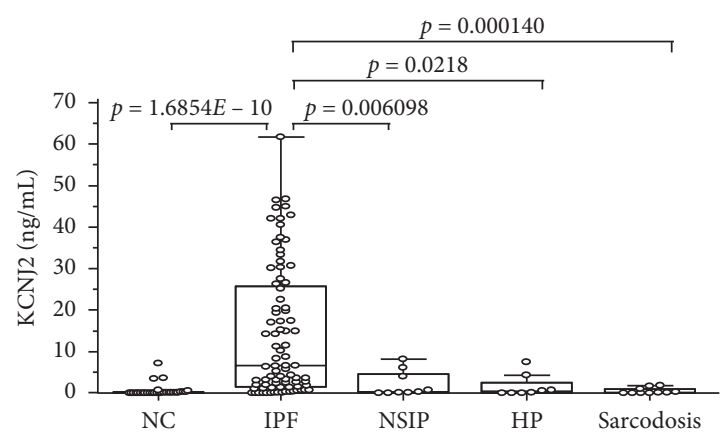

(a)

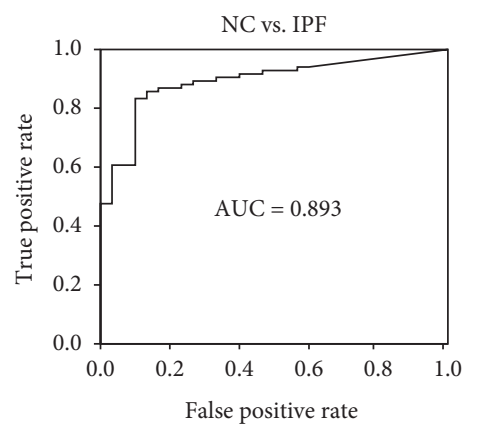

(b)

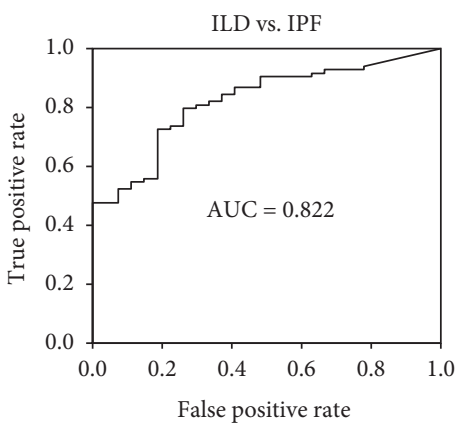

(c)

FIGURE 2: KCNJ2 protein concentrations in BAL fluids and ROC analysis. (a) KCNJ2 protein was detected in 18 of $30 \mathrm{NCs}, 79$ of 84 IPF patients, 7 of 9 NSIP patients, 5 of $8 \mathrm{HP}$ patients, and 9 of 10 sarcoidosis patients. The data are presented as median values with $25 \%$ and $75 \%$ quartiles. (b) ROC curve of the KCNJ2 protein levels in IPF patients and NCs. A cut-off value of $0.636 \mathrm{ng} / \mathrm{mL}$ had an AUC of 0.893 , a specificity of $90.0 \%$, and a sensitivity of $83.3 \%$ for differentiating IPF patients from NCs. (c) ROC curve of the KCNJ2 protein level in patients with IPF and those with other interstitial lung diseases.

other cell types are the major source of KCNJ2 expression. $\mathrm{K}^{+}$currents appear to be essential for the membrane functions of oligodendrocytes, astrocytes, and fibroblasts as well (18-21), even though KCNJ2 is expressed mainly in skeletal and cardiac muscle cells. Lung fibroblasts may express KCNJ2 upon activation, as in chronic inflammation and fibrosis in IPF, but this is still uncertain. Additionally, KCNJ2 is localized mainly near the plasma membrane [39]. Thus, KCNJ2 proteins were easily detected in BAL fluid in the present study.

KCNJ2 is associated with the gene ontology categories of G-protein-activated inward rectifier potassium channel activity and regulation of $\mathrm{K}^{+}$flow. Although the ionic basis of the membrane potential of fibroblasts in IPF has not been fully elucidated, KCNJ2 is thought to affect the proliferation and membrane functions of these cells as well. This is supported by the finding that alterations in membrane potential by $\mathrm{K}^{+}$currents modulated both the proliferation and morphology of cardiac myofibroblasts [19] in that exposure to low extracellular $\mathrm{K}^{+}$concentrations induced hyperpolarization and enhanced myofibroblast proliferation and survival, whereas exposure to high concentrations enhanced myofibroblast contractility, according to collagen I gel deformation assays [19].

In noncardiac fibroblasts and myofibroblasts, $\mathrm{K}^{+}$currents appear to be essential for maintaining the cellular functions of astrocytes [20], oligodendrocytes [23], and fibroblasts [22]. In each of these cell types, blocking $\mathrm{K}^{+}$currents inhibited proliferation [21]. Depolarization resulted in enhanced ventricular myofibroblast contractility according to enhanced collagen I gel deformation upon an increase in $\mathrm{K}^{+}$concentrations [19]. KCNJ2 expression mainly in cardiac muscle cells suggests a role for this protein in the development of Andersen syndrome and atrial fibrillation. Furthermore, atrial arrhythmias, including atrial fibrillation and atrial flutter, are the most common arrhythmias observed in patients with IPF [40]. The postulated mechanisms for arrhythmia development in these patients include the presence of hypoxia and chronic inflammation, elevated pulmonary pressure, and a risk of coronary artery disease [41]. However, genetic variants of KCNJ2 may also contribute to the high incidence of atrial arrhythmias in IPF patients. Thus, we assessed the arrhythmia incidence in our 84 study subjects using electrocardiography $(n=80)$ and Holter monitoring $(n=5)$. Among them, six subjects had atrial fibrillation or flutter. There was no difference in the KCNJ2 genotype frequency or BALF protein level between those with and those without atrial arrhythmia (Supplemental Table 1 and Supplemental Figure 2). Additionally, there was no significant difference in KCNJ2 expression according to sex or smoking status in the post hoc analysis (Supplemental Figures 1(b) and 1(c)). As a potential limitation of the present study, we did not evaluate the presence of hypoxia or pulmonary hypertension in the association analysis of atrial arrhythmias due to the small number of study subjects. In conclusion, KCNJ2 may be an indicator of the development of IPF and a surrogate marker for IPF differential diagnosis.

\section{Abbreviations}

IPF: Idiopathic pulmonary fibrosis

ECM: Extracellular matrix

BAL: Bronchoalveolar lavage

NC: Normal controls

NSIP: Nonspecific interstitial pneumonia

HP: Hypersensitivity pneumonitis

ROC: Receiver operating characteristic

IFN: Interferon.

\section{Data Availability}

The data used to support the findings of this study are available from the corresponding author upon request.

\section{Disclosure}

None of the authors has a financial relationship with a commercial entity that has an interest in the subject of this manuscript. 


\section{Conflicts of Interest}

The authors declare that they have no conflicts of interest.

\section{Authors' Contributions}

JU Lee and CS Park conceived and designed the experiments; CA Jung and RH Kim performed the fibroblast culture and RNA isolation; JU Lee performed ELISA; CS Park provided clinical samples; HS Chang performed statistical analysis; JU Lee and JS Park wrote the manuscript.

\section{Acknowledgments}

This study was supported by a grant from the National Research Foundation of Korea (NRF) funded by the Ministry of Education (2017R1D1A1B0429365) and a research grant from Soonchunhyang University to JS Park. JU Lee was supported by the Basic Science Research Program of NRF (2018R1A6A3A01011004). The samples were generously provided by a Biobank in Soonchunhyang University, Bucheon Hospital.

\section{Supplementary Materials}

Supplemental Figure 1: KCNJ2 protein concentrations in BAL fluids from IPF patients according to the GAP stage, smoking, and sex. Comparisons of KCNJ2 protein levels according to (a) the GAP stage, (b) smoking status, and (c) sex. Supplemental Figure 2: KCNJ2 protein concentrations in BAL fluids from IPF patients with or without atrial arrhythmias. Supplemental Table 1: comparison with genotype frequency of KCNJ2 in subjects with idiopathic pulmonary fibrosis with or without atrial arrhythmia. (Supplementary Materials)

\section{References}

[1] A. T. Society, "Idiopathic pulmonary fibrosis, diagnosis and treatment.(International consensus statement)," American Journal of Respiratory and Critical Care Medicine, vol. 161, pp. 646-664, 2000.

[2] D. S. Kim, H. R. Collard, and T. E. King Jr., "Classification and natural history of the idiopathic interstitial pneumonias," Proceedings of the American Thoracic Society, vol. 3, no. 4, pp. 285-292, 2006.

[3] T. A. Wynn, "Integrating mechanisms of pulmonary fibrosis," The Journal of Experimental Medicine, vol. 208, no. 7, pp. 1339-1350, 2011.

[4] M. Wahidi, D. Schwartz, and G. Raghu, "Genetics of familial pulmonary fibrosis and other variants," Lung Biology in Health and Disease, vol. 185, pp. 31-54, 2004.

[5] D. A. Schwartz, "Idiopathic pulmonary fibrosis is a complex genetic disorder," Transactions of the American Clinical and Climatological Association, vol. 127, no. 127, pp. 34-45, 2016.

[6] K. Boon, N. W. Bailey, J. Yang et al., "Molecular phenotypes distinguish patients with relatively stable from progressive idiopathic pulmonary fibrosis (IPF)," PloS One, vol. 4, no. 4, p. e5134, 2009.

[7] K. Konishi, K. F. Gibson, K. O. Lindell et al., "Gene expression profiles of acute exacerbations of idiopathic pulmonary fibrosis," American Journal of Respiratory and Critical Care Medicine, vol. 180, no. 2, pp. 167-175, 2009.

[8] M. Selman, A. Pardo, L. Barrera et al., "Gene expression profiles distinguish idiopathic pulmonary fibrosis from hypersensitivity pneumonitis," American Journal of Respiratory and Critical Care Medicine, vol. 173, no. 2, pp. 188-198, 2006.

[9] F. Zuo, N. Kaminski, E. Eugui et al., "Gene expression analysis reveals matrilysin as a key regulator of pulmonary fibrosis in mice and humans," Proceedings of the National Academy of Sciences, vol. 99, no. 9, pp. 6292-6297, 2002.

[10] J.-U. Lee, H. S. Cheong, E.-Y. Shim et al., "Gene profile of fibroblasts identify relation of CCL8 with idiopathic pulmonary fibrosis," Respiratory Research, vol. 18, no. 1, p. 3, 2017.

[11] Z. Lu and R. MacKinnon, "Electrostatic tuning of $\mathrm{Mg}^{2+}$ affinity in an inward-rectifier $\mathrm{K}^{+}$channel," Nature, vol. 371, no. 6494, pp. 243-246, 1994.

[12] H.-K. Chang and R.-C. Shieh, "Voltage-dependent inhibition of outward Kir2.1 currents by extracellular spermine," Biochimica et Biophysica Acta (BBA)-Biomembranes, vol. 1828, no. 2, pp. 765-775, 2013.

[13] H. T. Kurata, A. Akrouh, J. B. W. Li, L. J. Marton, and C. G. Nichols, "Scanning the topography of polyamine blocker binding in an inwardly rectifying potassium channel," Journal of Biological Chemistry, vol. 288, no. 9, pp. 6591-6601, 2013.

[14] B. Fakler, U. Brändle, E. Glowatzki, S. Weidemann, H.-P. Zenner, and J. P. Ruppersberg, "Strong voltage-dependent inward rectification of inward rectifier $\mathrm{K}^{+}$channels is caused by intracellular spermine," Cell, vol. 80, no. 1, pp. 149-154, 1995.

[15] K. F. Raab-Graham, C. M. Radeke, and C. A. Vandenberg, "Molecular cloning and expression of a human heart inward rectifier potassium channel," Neuroreport, vol. 5, no. 18, pp. 2501-2505, 1994.

[16] H. Zhang, H. Cheng, X. Yan, T. Mirshahi, and D. E. Logothetis, "Activation of inwardly rectifying $\mathrm{K}^{+}$ channels by distinct PtdIns $(4,5)$ P 2 interactions," Nature Cell Biology, vol. 1, no. 3, 1999.

[17] Y. Kubo, T. J. Baldwin, Y. Nung Jan, and L. Y. Jan, "Primary structure and functional expression of a mouse inward rectifier potassium channel," Nature, vol. 362, no. 6416, pp. 127-133, 1993.

[18] X.-Y. Qi, H. Huang, B. Ordog et al., "Fibroblast inwardrectifier potassium current upregulation in profibrillatory atrial remodeling," Circulation Research, vol. 116, no. 5, pp. 836-845, 2015.

[19] L. Chilton, S. Ohya, D. Freed et al., " $\mathrm{K}^{+}$currents regulate the resting membrane potential, proliferation, and contractile responses in ventricular fibroblasts and myofibroblasts," American Journal of Physiology-Heart and Circulatory Physiology, vol. 288, no. 6, pp. H2931-H2939, 2005.

[20] C. A. Pappas, N. Ullrich, and H. Sontheimer, "Reduction of glial proliferation by $\mathrm{K}+$ channel blockers is mediated by changes in pHi," Neuroreport, vol. 6, no. 1, pp. 193-196, 1994.

[21] L. C. Schlichter, G. Sakellaropoulos, B. Ballyk, P. S. Pennefather, and D. J. Phipps, "Properties of $\mathrm{K}^{+}$and $\mathrm{Cl}^{-}$ channels and their involvement in proliferation of rat microglial cells," Glia, vol. 17, no. 3, pp. 225-236, 1996.

[22] T. L. Peña, S. H. Chen, S. F. Konieczny, and S. G. Rane, "Ras/ MEK/ERK up-regulation of the fibroblast KCaChannel FIK is a common mechanism for basic fibroblast growth factor and transforming growth factor- $\beta$ suppression of myogenesis," 
Journal of Biological Chemistry, vol. 275, no. 18, pp. 1367713682, 2000.

[23] P. Knutson, C. A. Ghiani, J.-M. Zhou, V. Gallo, and C. J. McBain, " $\mathrm{K}^{+}$Channel expression and cell proliferation are regulated by intracellular sodium and membrane depolarization in oligodendrocyte progenitor cells," The Journal of Neuroscience, vol. 17, no. 8, pp. 2669-2682, 1997.

[24] V. N. Bavro, R. De Zorzi, M. R. Schmidt et al., "Structure of a KirBac potassium channel with an open bundle crossing indicates a mechanism of channel gating," Nature Structural \& Molecular Biology, vol. 19, no. 2, pp. 158-163, 2012.

[25] S. B. Hansen, X. Tao, and R. MacKinnon, "Structural basis of PIP2 activation of the classical inward rectifier $\mathrm{K}^{+}$channel Kir2.2," Nature, vol. 477, no. 7365, pp. 495-498, 2011.

[26] L. Wollin, E. Wex, A. Pautsch et al., "Mode of action of nintedanib in the treatment of idiopathic pulmonary fibrosis," European Respiratory Journal, vol. 45, p. 1434, 2015.

[27] M. Selman, A. Pardo, and T. E. King Jr., "Hypersensitivity pneumonitis," American Journal of Respiratory and Critical Care Medicine, vol. 186, no. 4, pp. 314-324, 2012.

[28] S. Parrish and J. F. Turner, "Diagnosis of sarcoidosis," Diseasea-Month, vol. 55, no. 11, pp. 693-703, 2009.

[29] R. P. Baughman, D. A. Culver, and M. A. Judson, "A concise review of pulmonary sarcoidosis," American Journal of Respiratory and Critical Care Medicine, vol. 183, no. 5, pp. 573-581, 2011.

[30] W. D. Travis, U. Costabel, D. M. Hansell et al., "An official American Thoracic Society/European Respiratory Society statement: update of the international multidisciplinary classification of the idiopathic interstitial pneumonias," American Journal of Respiratory and Critical Care Medicine, vol. 188, no. 6, pp. 733-748, 2013.

[31] A. T. Society, "This joint statement of the American thoracic society (ATS), and the European respiratory society (ERS) was adopted by the ATS board of directors, june 2001 and by the ERS executive committee, june 2001," American journal of respiratory and critical care medicine, vol. 165, pp. 277-304, 2002.

[32] W. D. Travis, G. Hunninghake, T. E. King et al., "Idiopathic nonspecific interstitial pneumonia," American Journal of Respiratory and Critical Care Medicine, vol. 177, no. 12, pp. 1338-1347, 2008.

[33] B. Ley, C. J. Ryerson, E. Vittinghoff et al., "A multidimensional index and staging system for idiopathic pulmonary fibrosis," Annals of Internal Medicine, vol. 156, no. 10, pp. 684-691, 2012.

[34] G. Raghu, Y. Chen, V. Rusch, and P. S. Rabinovitch, "Differential proliferation of fibroblasts cultured from normal and fibrotic human lungs," American Review of Respiratory Disease, vol. 138, no. 3, pp. 703-708, 1988.

[35] K. J. Livak and T. D. Schmittgen, "Analysis of relative gene expression data using real-time quantitative PCR and the $2^{-\Delta \Delta \mathrm{CT}}$ method," Methods, vol. 25, no. 4, pp. 402-408, 2001.

[36] K. K. Kim, M. C. Kugler, P. J. Wolters et al., "Alveolar epithelial cell mesenchymal transition develops in vivo during pulmonary fibrosis and is regulated by the extracellular matrix," Proceedings of the National Academy of Sciences, vol. 103, no. 35, pp. 13180-13185, 2006.

[37] T. H. Kim, Y. H. Lee, K. H. Kim et al., "Role of lung apolipoprotein A-I in idiopathic pulmonary fibrosis," American Journal of Respiratory and Critical Care Medicine, vol. 182, no. 5, pp. 633-642, 2010.

[38] S.-W. Park, M.-H. Ahn, H. K. Jang et al., "Interleukin-13 and its receptors in idiopathic interstitial pneumonia: clinical implications for lung function," Journal of Korean Medical Science, vol. 24, no. 4, pp. 614-620, 2009.

[39] Y. Hosaka, H. Hanawa, T. Washizuka et al., "Function, subcellular localization and assembly of a novel mutation of KCNJ2 in Andersen's syndrome," Journal of Molecular and Cellular Cardiology, vol. 35, no. 4, pp. 409-415, 2003.

[40] Y. Shibata, T. Watanabe, D. Osaka et al., "Impairment of pulmonary function is an independent risk factor for atrial fibrillation: the Takahata study," International Journal of Medical Sciences, vol. 8, no. 7, pp. 514-522, 2011.

[41] S. Patanè, F. Marte, M. Sturiale, G. Dattilo, and F. Luzza, "Atrial flutter, ventricular tachycardia and changing axis deviation associated with scleroderma," International Journal of Cardiology, vol. 153, no. 2, pp. e25-e28, 2011. 